$$
Y / \angle B--15968
$$

\title{
Y-12
}

Report No. Y/LB-15,968

\section{OAK RIDGE \\ $\mathrm{Y}-12$ \\ PLANT}

\section{NWIS CASTING MEASUREMENTS TAKEN DURING DEMONSTRATIONS TO RUSSIAN VISITORS}

\author{
J. A. Mullens \\ T. E. Valentine \\ J. T. Mihalczo
}

\section{RECEIVED
JUN 089989 \\ OSTI}

MANAGED BY

LOCKHEED MARTIN ENERGY SYSTEMS, INC. FOR THE UNITED STATES

DEPARTMENT OF ENERGY
April 15, 1998

Nuclear Materials Management and Storage Program Office

\section{April 15, 1998}

Prepared by the

Oak Ridge Y-12 Plant

Oak Ridge, Tennessee 37831 managed by

Lockheed Martin Energy Systems, Inc. for the

U.S. DEPARTMENT OF ENERGY

under contract DE-AC05-84OR21400

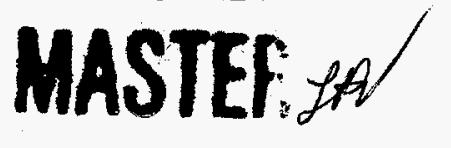




\section{DISCLAIMER}

This report was prepared as an account of work sponsored by an agency of the United States government. Neither the United States Government nor any agency thereof, nor any of their employees, makes any warranty, express or implied, or assumes any legal liability or responsibility for the accuracy, completeness, or usefulness of any information, apparatus, product, or process disclosed, or represents that its use would not infringe privately owned rights. Reference herein to any specific commercial product, process, or service by trade name, trademark, manufacturer, or otherwise, does not necessarily constitute or imply its endorsement, recommendation, or favoring by the United States Government or any agency thereof. The views and opinions of authors expressed herein do not necessarily state or reflect those of the United States Government or any agency thereof.

\section{COPYRIGHT NOTICE}

The submitted manuscript has been authored by a contractor of the U.S. Government under contract DE-AC05-84OR21400. Accordingly, the U.S. Government retains a paid-up, nonexclusive, irrevocable, worldwide license to publish or reproduce the published form of this contribution, prepare derivative works, distribute copies to the public, and perform publicly and display publicly, or allow others to do so, for U.S. Government purposes. 


\section{DISCLAIMER}

Portions of this document may be illegible electronic image products. Images are produced from the best available original document. 
NWIS Casting Measurements Taken During Demonstrations to Russian Visitors

This report describes a set of NWIS measurements made during demonstrations to Russian visitors on August 28, 1997. These measurements will be given to the Russian visitors from Arzamus-16 as part of their NWIS training (part of a DOE laboratory-to-laboratory exchange program).

These measurements are made on standard highly enriched Uranium annular castings (as used for storage). Associated NWIS calibration runs were made in air (no casting, just the NWIS Californium source and detectors).

\section{NWIS Casting Measurements}

Table 1 lists the casting measurements in this set. Each measurement contains data from the ${ }^{252} \mathrm{Cf}$ detector and two detectors. The Uranium casting was between the Cf source and the other detectors.

Table 1 Casting Measurements for Aug 28, 1997 NWIS demonstration.

$\begin{array}{lrrrrrrr}\text { Name } & \text { channels } & \begin{array}{c}\text { sample } \\ \text { rate }(\mathrm{MHz})\end{array} & \text { block size } & \text { blocks } & \begin{array}{c}\text { Det1-Cf } \\ \text { (cps) }\end{array} & \begin{array}{l}\text { Det2 } \\ \text { (cps) }\end{array} & \begin{array}{l}\text { Det3 } \\ \text { (cps) }\end{array} \\ \text { Cast2801 } & 3 & 1000 & 512 & 1.00 \mathrm{E}+08 & 430469 & 14213 & 9155 \\ \text { Cast2802 } & 3 & 1000 & 512 & 1.00 \mathrm{E}+08 & 430527 & 13337 & 9127 \\ \text { Cast2803 } & 3 & 1000 & 512 & 1.00 \mathrm{E}+08 & 430527 & 13767 & 9304 \\ \text { Cast2804 } & 3 & 1000 & 512 & 1.00 \mathrm{E}+08 & 430625 & 14030 & 9404 \\ \text { Cast2805 } & 3 & 1000 & 512 & 1.00 \mathrm{E}+08 & 430605 & 13859 & 9013 \\ \text { Cast2806 } & 3 & 1000 & 512 & 1.00 \mathrm{E}+08 & 430703 & 14778 & 9433 \\ \text { Cast2807 } & 3 & 1000 & 512 & 1.00 \mathrm{E}+08 & 430508 & 15052 & 9384 \\ \text { Cast2808 } & 3 & 1000 & 512 & 1.00 \mathrm{E}+07 & 430840 & 13049 & 8525 \\ \text { Cast2809 } & 3 & 1000 & 512 & 1.00 \mathrm{E}+07 & 430977 & 13779 & 8955 \\ \text { Cast2810 } & 3 & 1000 & 512 & 1.00 \mathrm{E}+07 & 430352 & 14533 & 9244 \\ \text { Cast2811 } & 3 & 1000 & 512 & 1.00 \mathrm{E}+07 & 430391 & 13564 & 8827 \\ \text { Cast2812 } & 3 & 1000 & 512 & 1.00 \mathrm{E}+07 & 431035 & 15272 & 9416 \\ \text { Cast2813 } & 3 & 1000 & 512 & 1.00 \mathrm{E}+07 & 430234 & 13561 & 8612 \\ \text { Cast2814 } & 3 & 1000 & 512 & 1.00 \mathrm{E}+07 & 430781 & 13885 & 8907 \\ \text { Cast2815 } & 3 & 1000 & 512 & 1.00 \mathrm{E}+09 & 430547 & 13409 & 8782 \\ \text { Cast2816 } & 3 & 1000 & 512 & 1.00 \mathrm{E}+08 & 430449 & 12956 & 8887\end{array}$

Figures 1 to 27 show the measurement signatures for these measurements.

\section{NWIS Calibration Measurements}

Table 2 lists the calibration measurements in this set. These measurements were made with the same detectors as the casting measurements above.

Table 2 Calibration measurements for Aug 28, 1997 NWIS demonstration.

\begin{tabular}{rrrrrrrr} 
Name & channels & $\begin{array}{c}\text { sample } \\
\text { rate }(\mathrm{MHz})\end{array}$ & block size & blocks & $\begin{array}{c}\text { Det1 }-\mathrm{Cf} \\
\text { (cps) }\end{array}$ & $\begin{array}{l}\text { Det2 } \\
\text { (cps) }\end{array}$ & \multicolumn{1}{l}{$\begin{array}{l}\text { Det3 } \\
\text { (cps) }\end{array}$} \\
cal2801 & 3 & 1000 & 512 & $1.00 \mathrm{E}+08$ & 430664 & 26531 & 19396 \\
cal2802 & 3 & 1000 & 512 & $1.00 \mathrm{E}+09$ & 430547 & 26648 & 19370 \\
cal2803 & 3 & 1000 & 512 & $1.00 \mathrm{E}+08$ & 430703 & 25266 & 19629
\end{tabular}


Graphic 7. 0 Apr. 14, 1998 1:11:00 PM

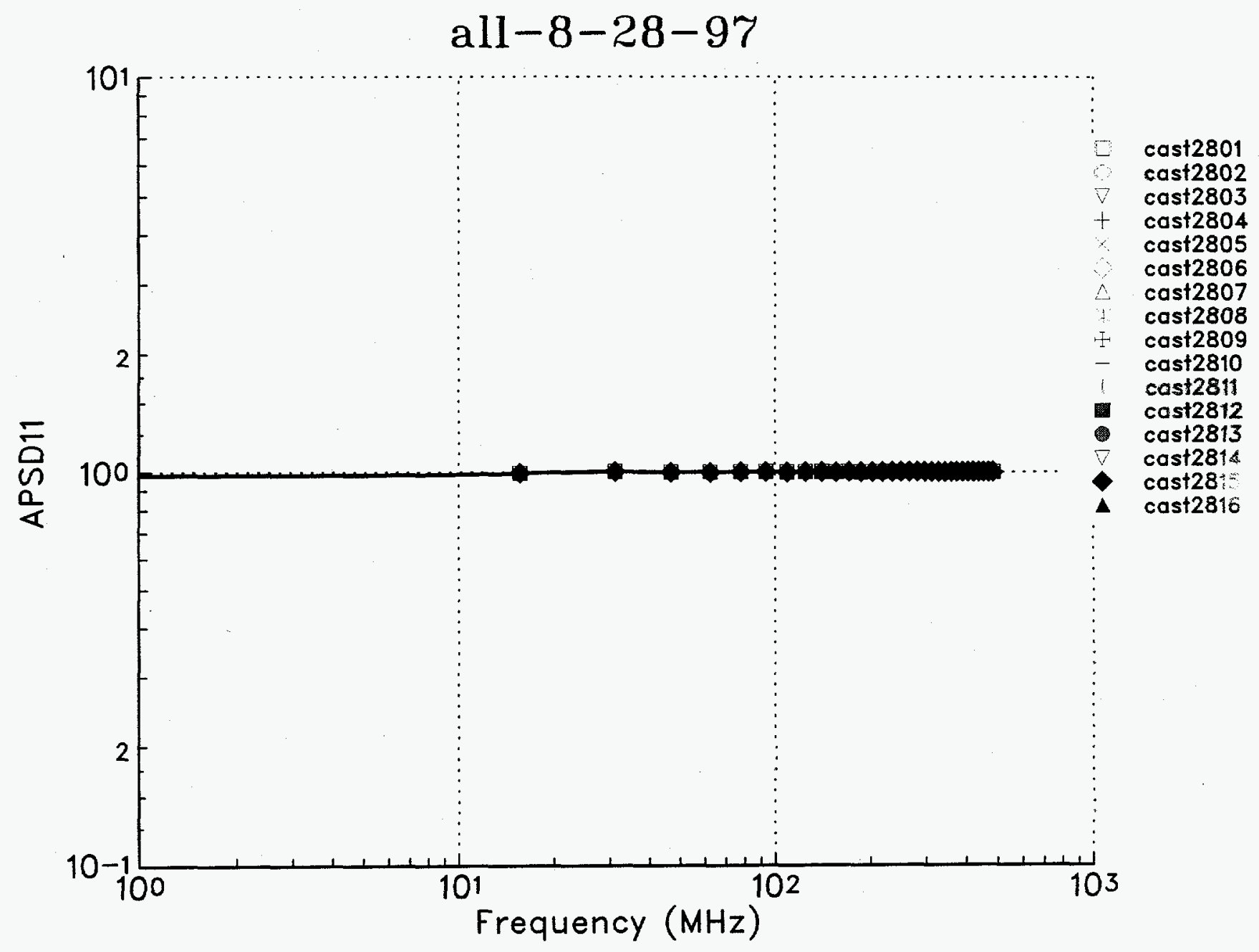

(1) 


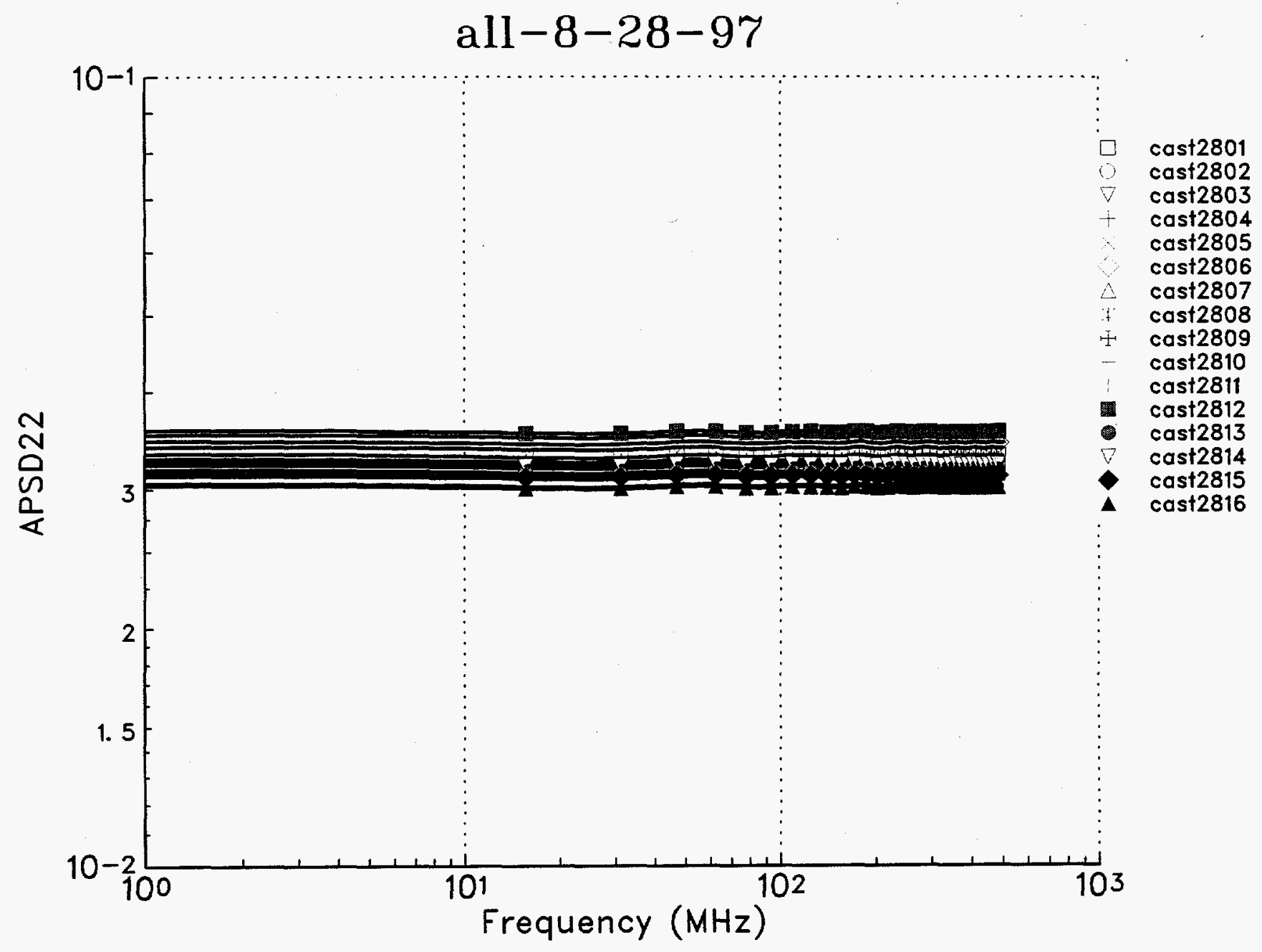

(2) 
Graphic 7.0 Apr. 14, 1998 1:13:15 PM

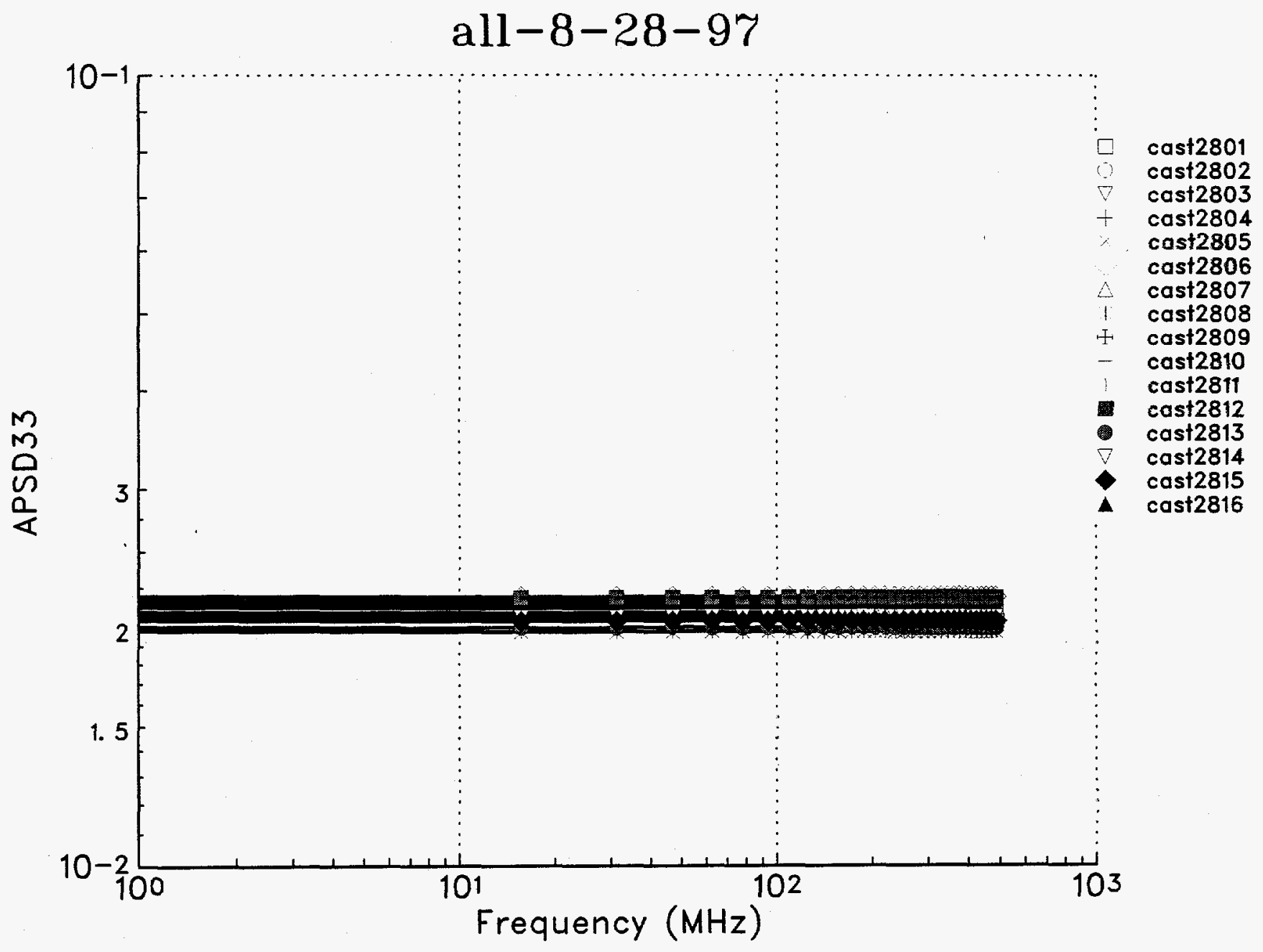

(3) 


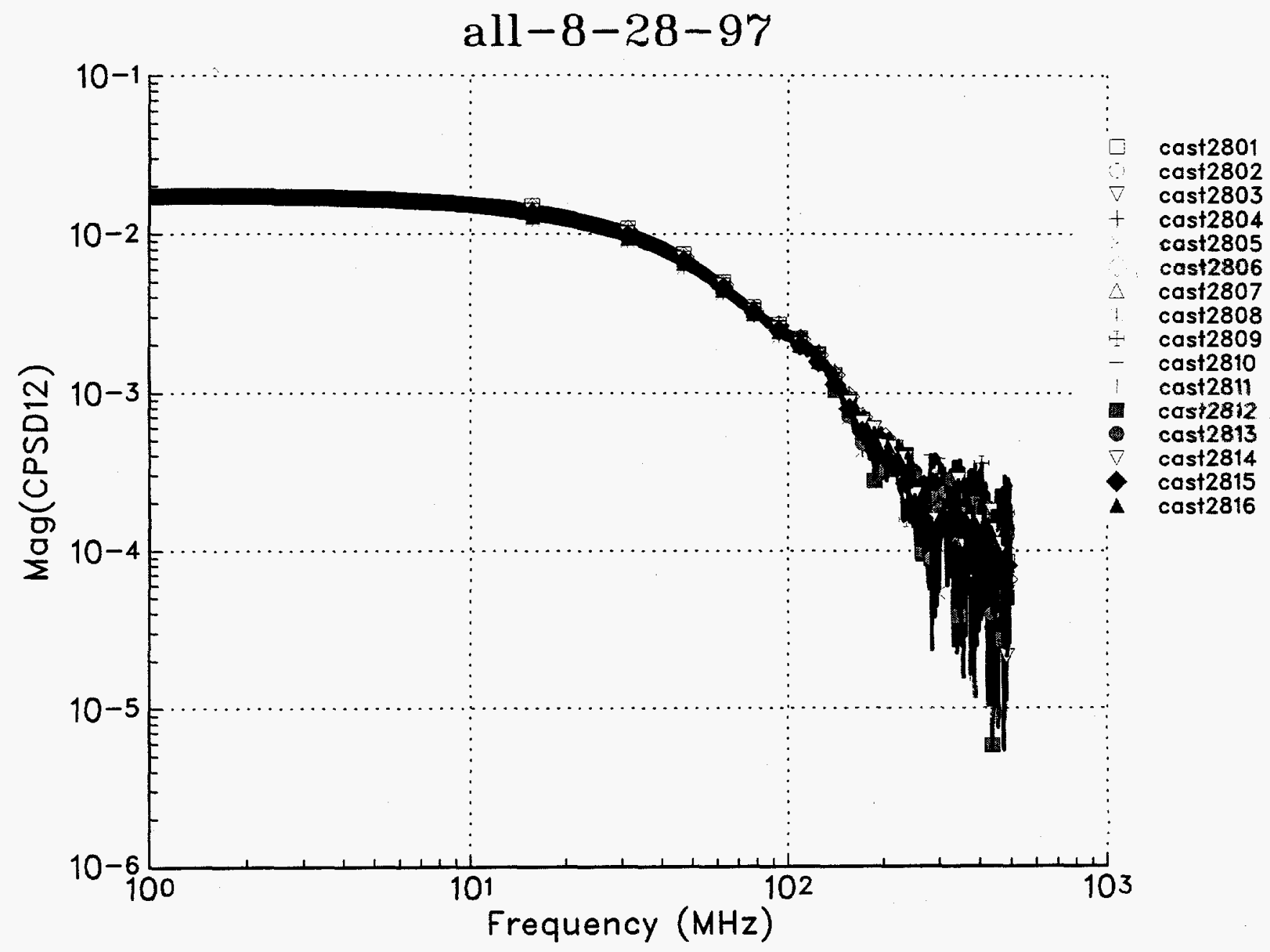

(4) 
Graphic 7.0 Apr. 14, 1998 1:18:06 PM

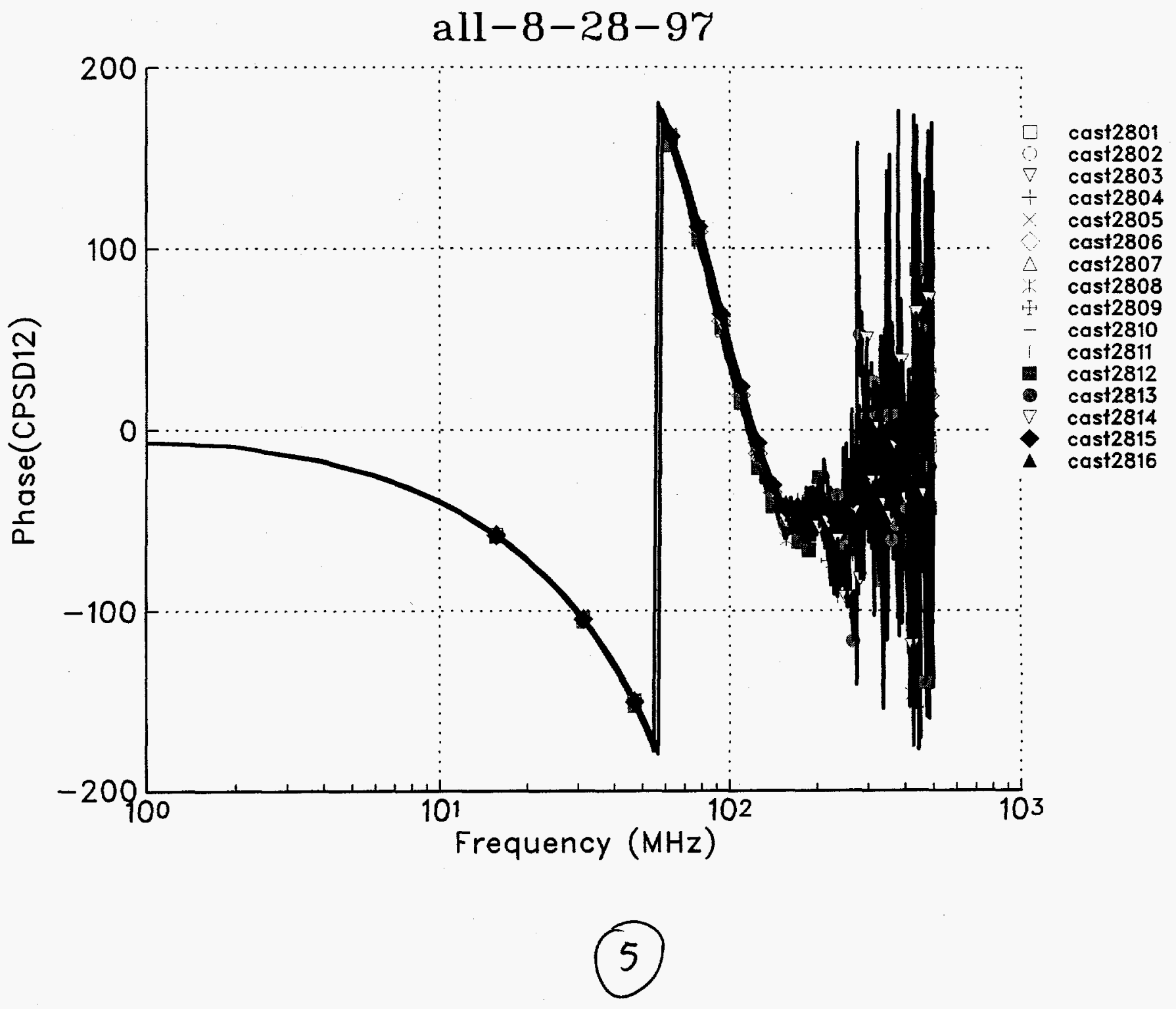




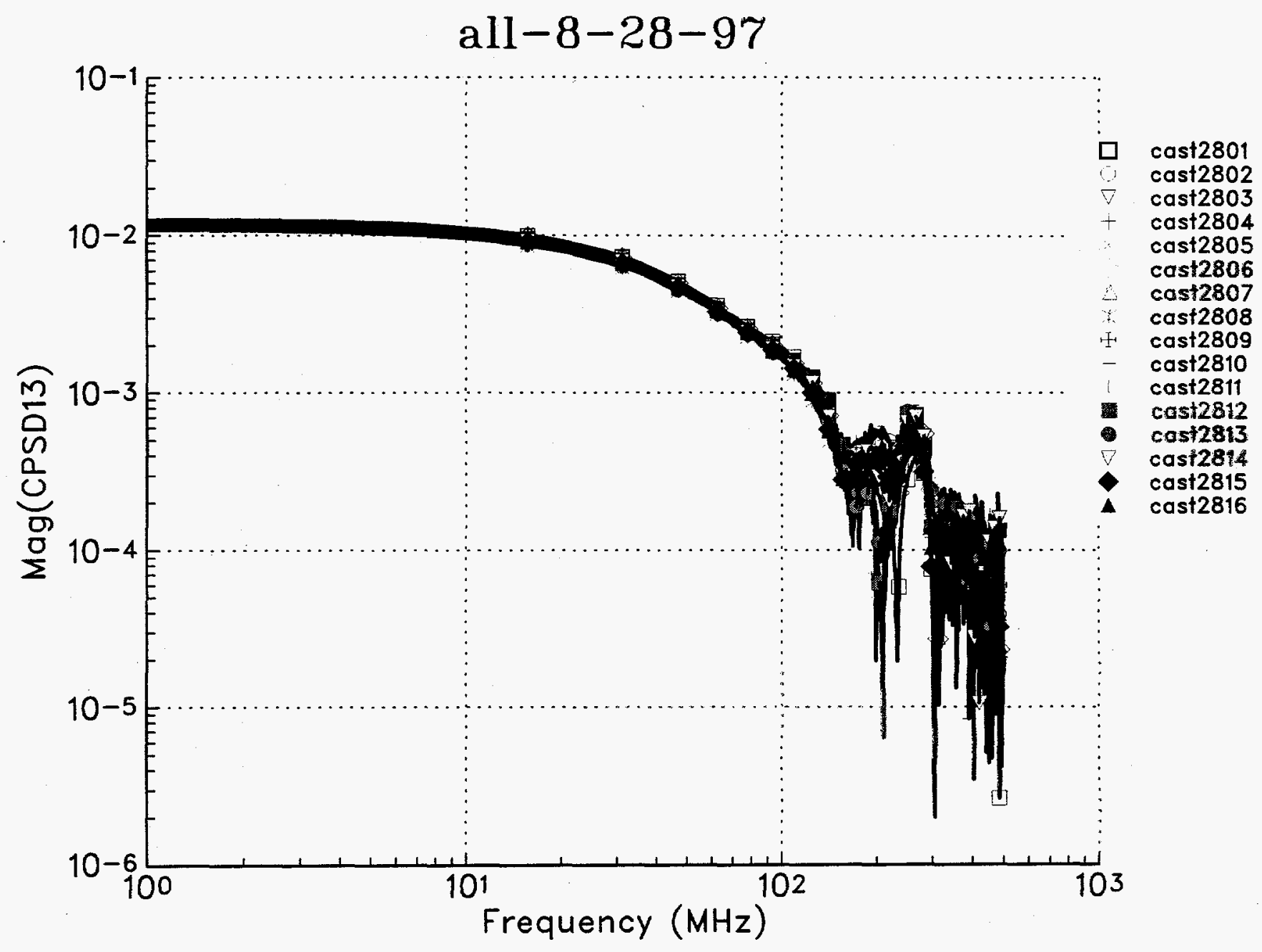

(b) 
Graphic 7. 0 Apr. 14, 1998 1:22:35 PM

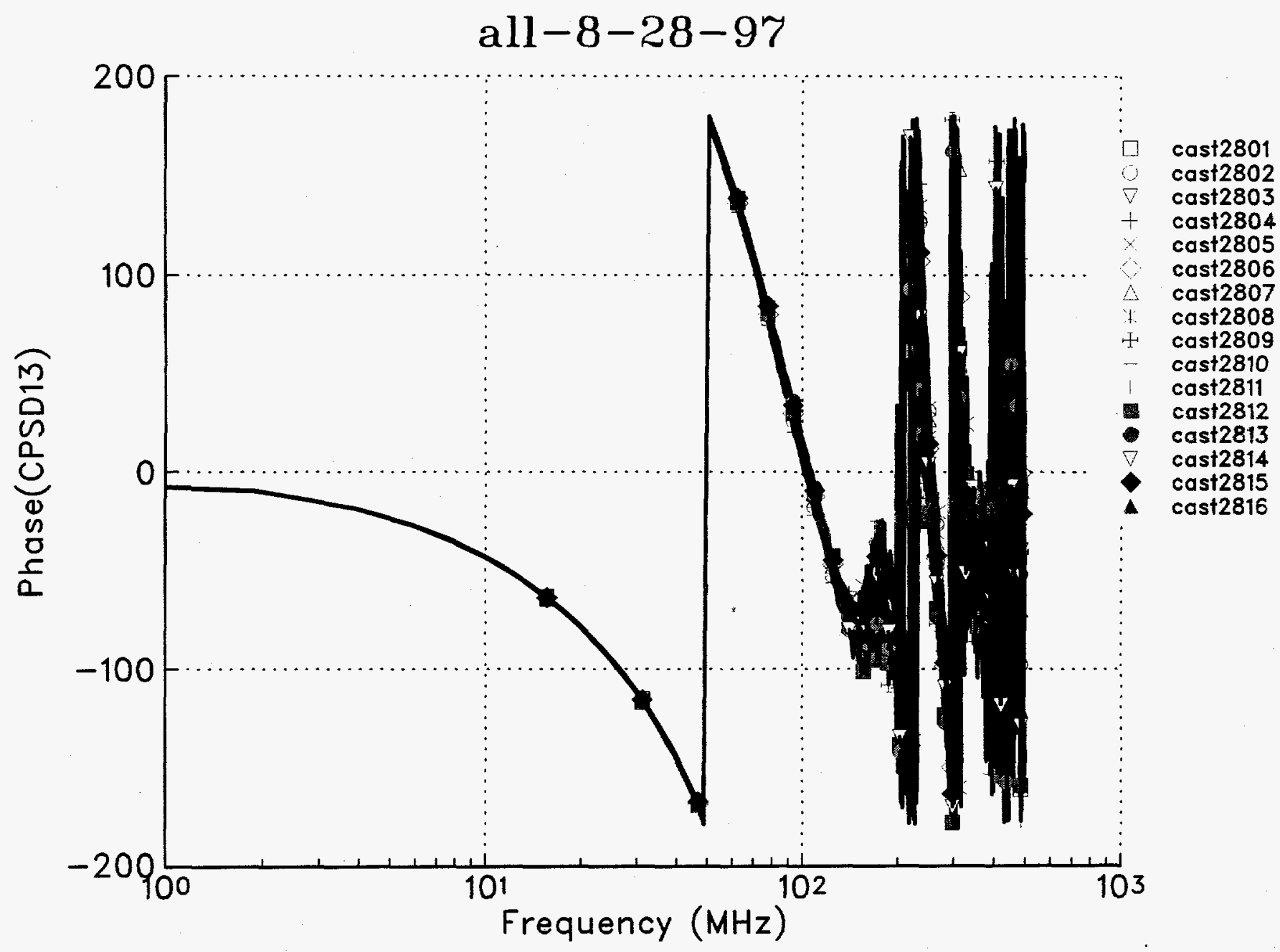




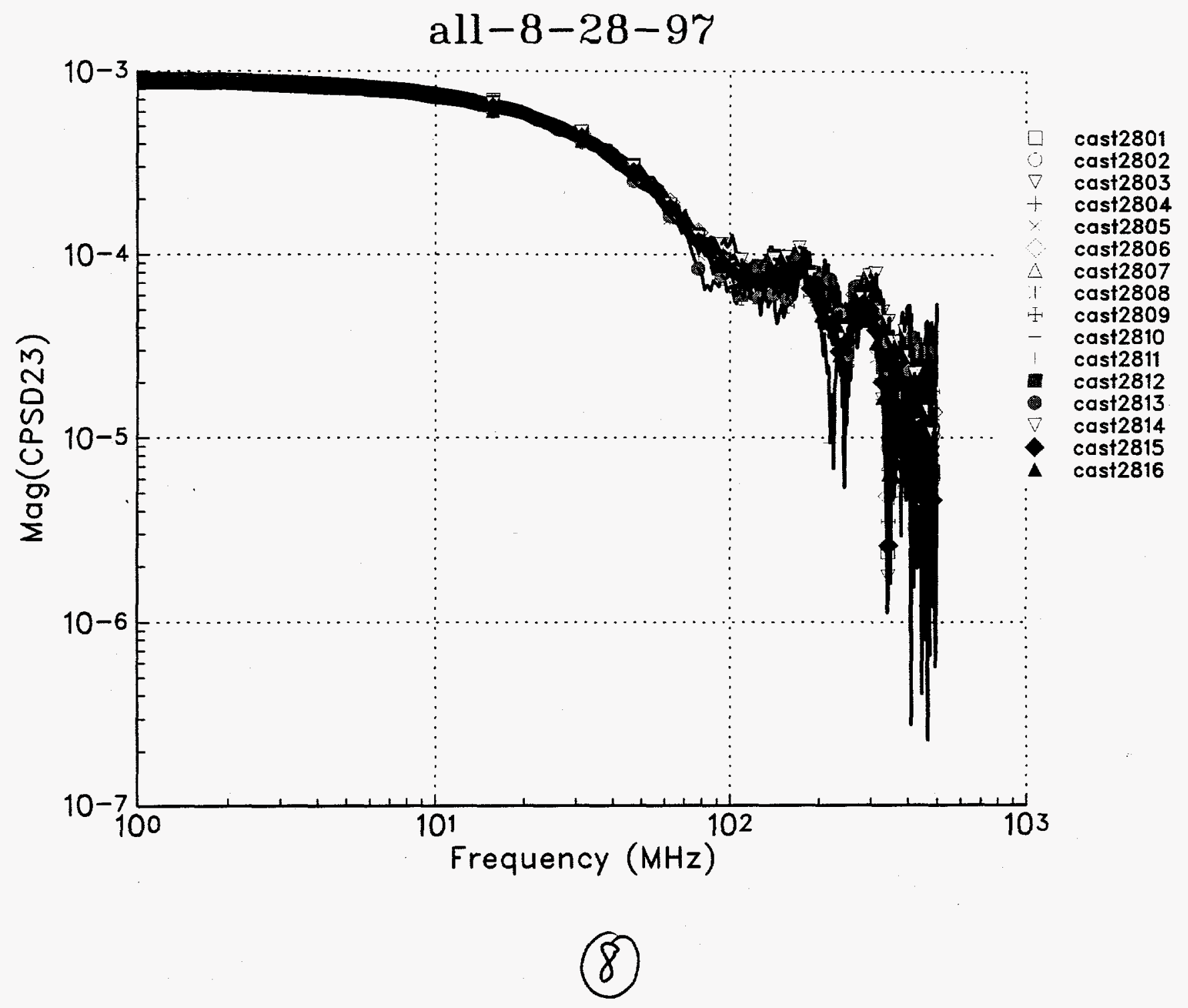


Grophic 7.0 Apr. 14, 1998 1:20:42 PM

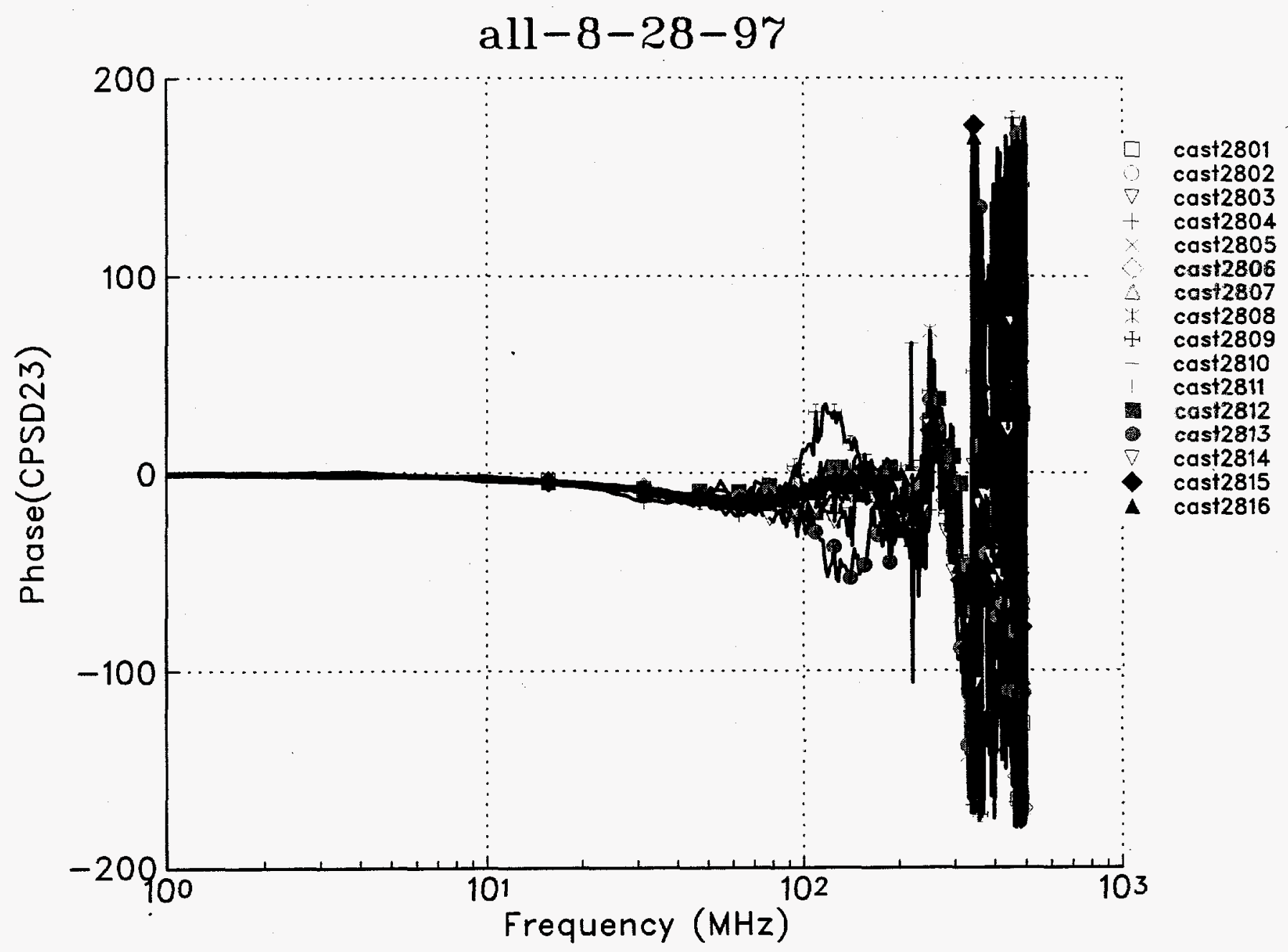

(9) 


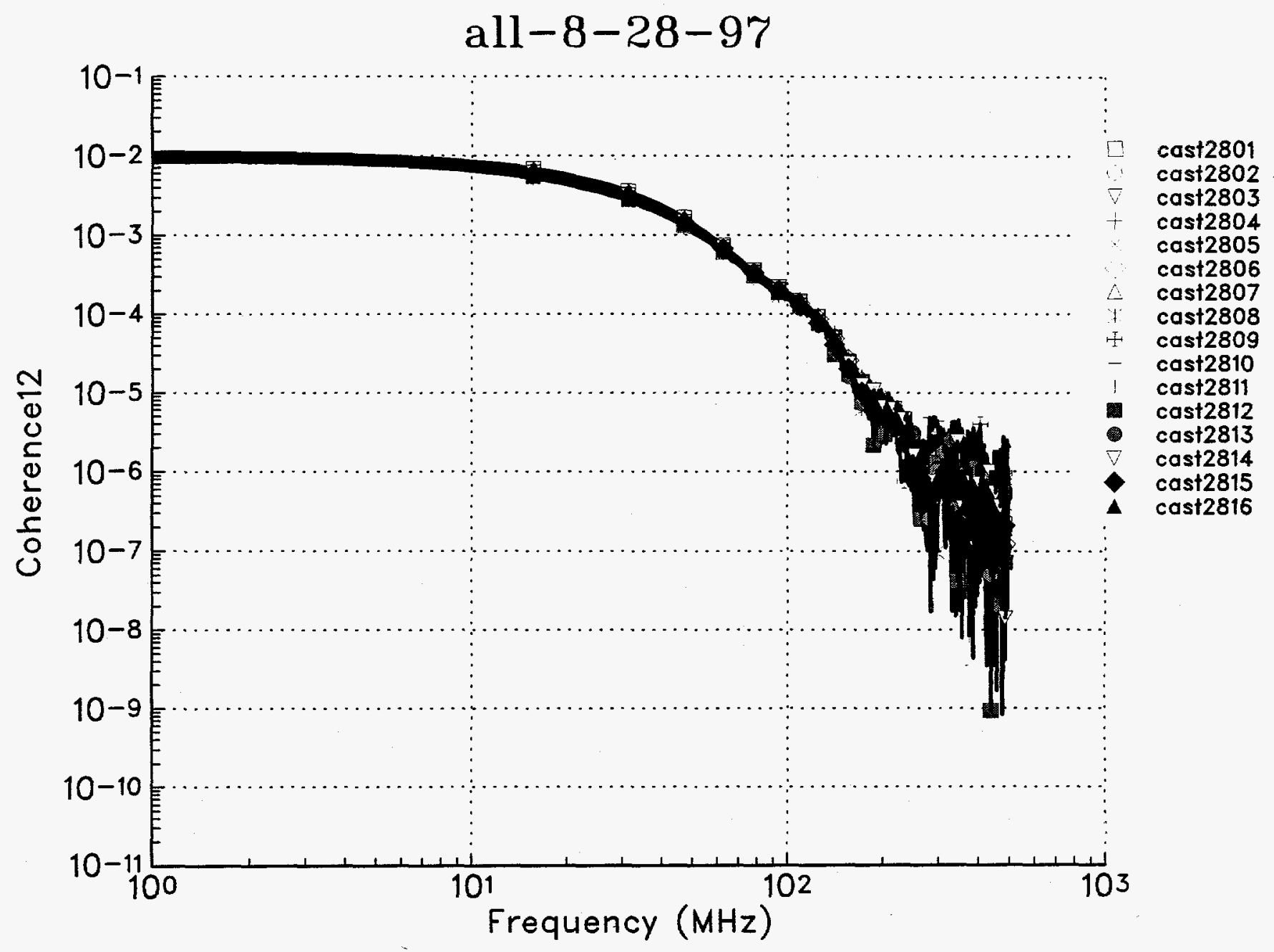

(10) 


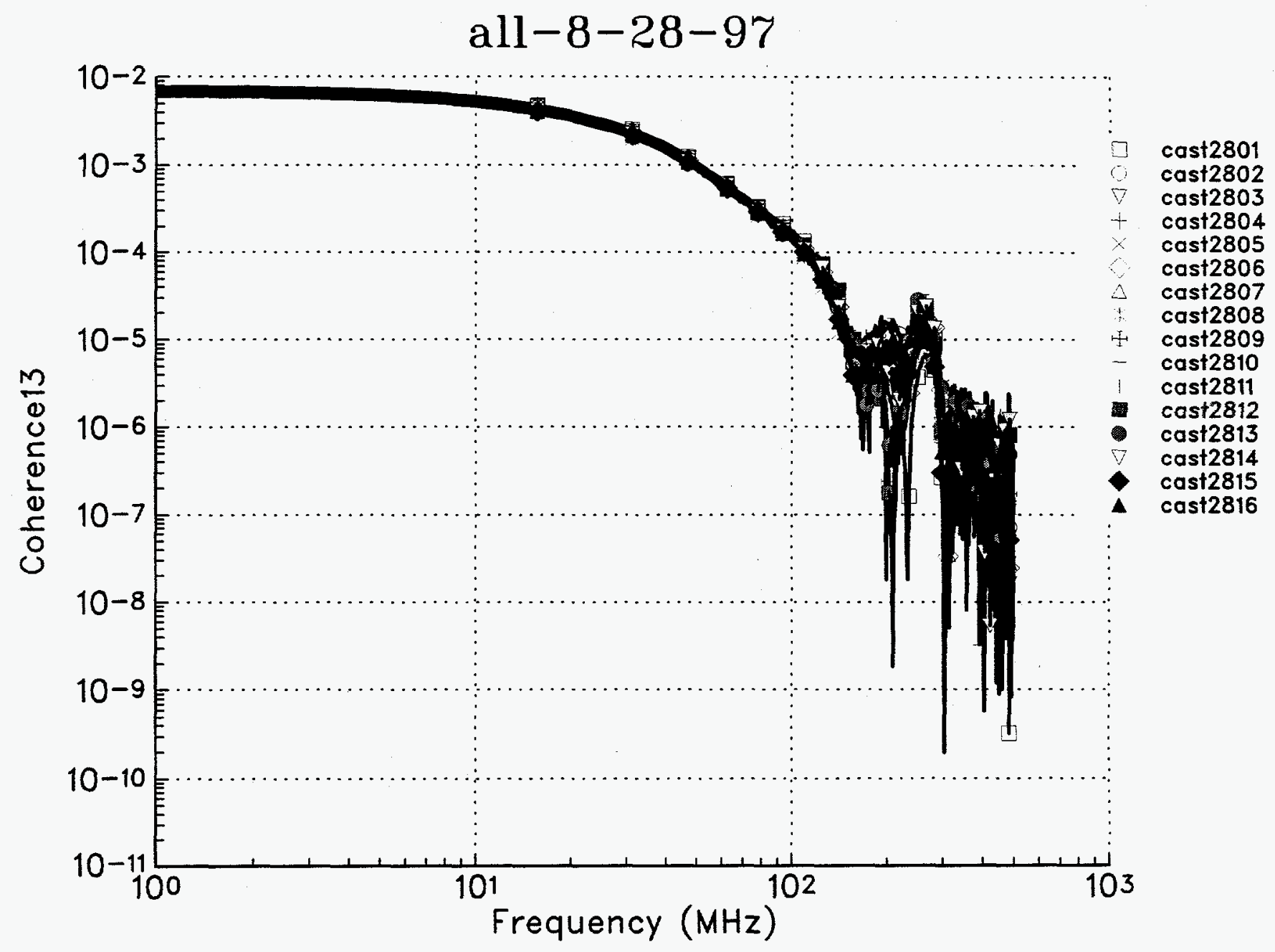

(i1) 


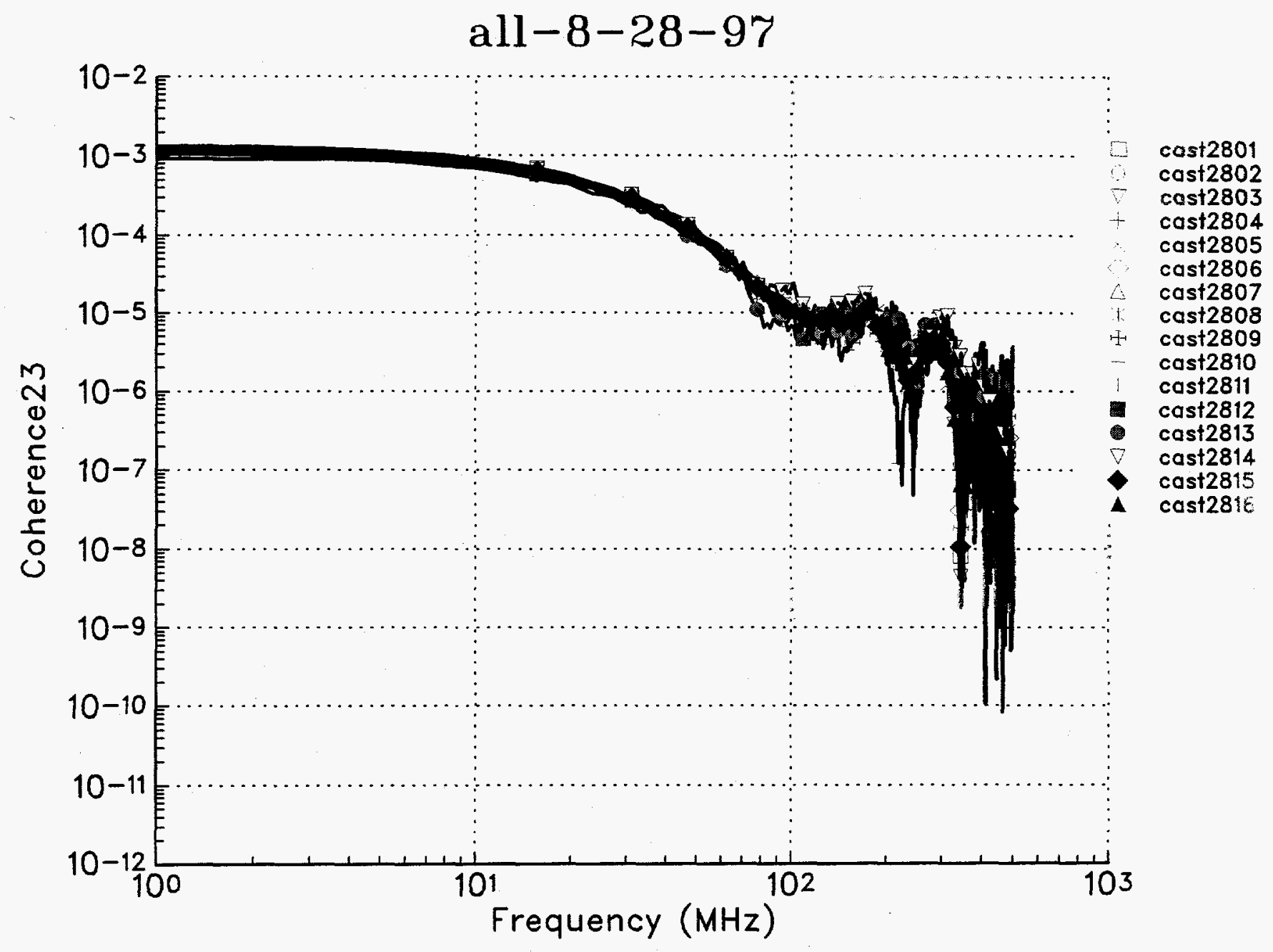




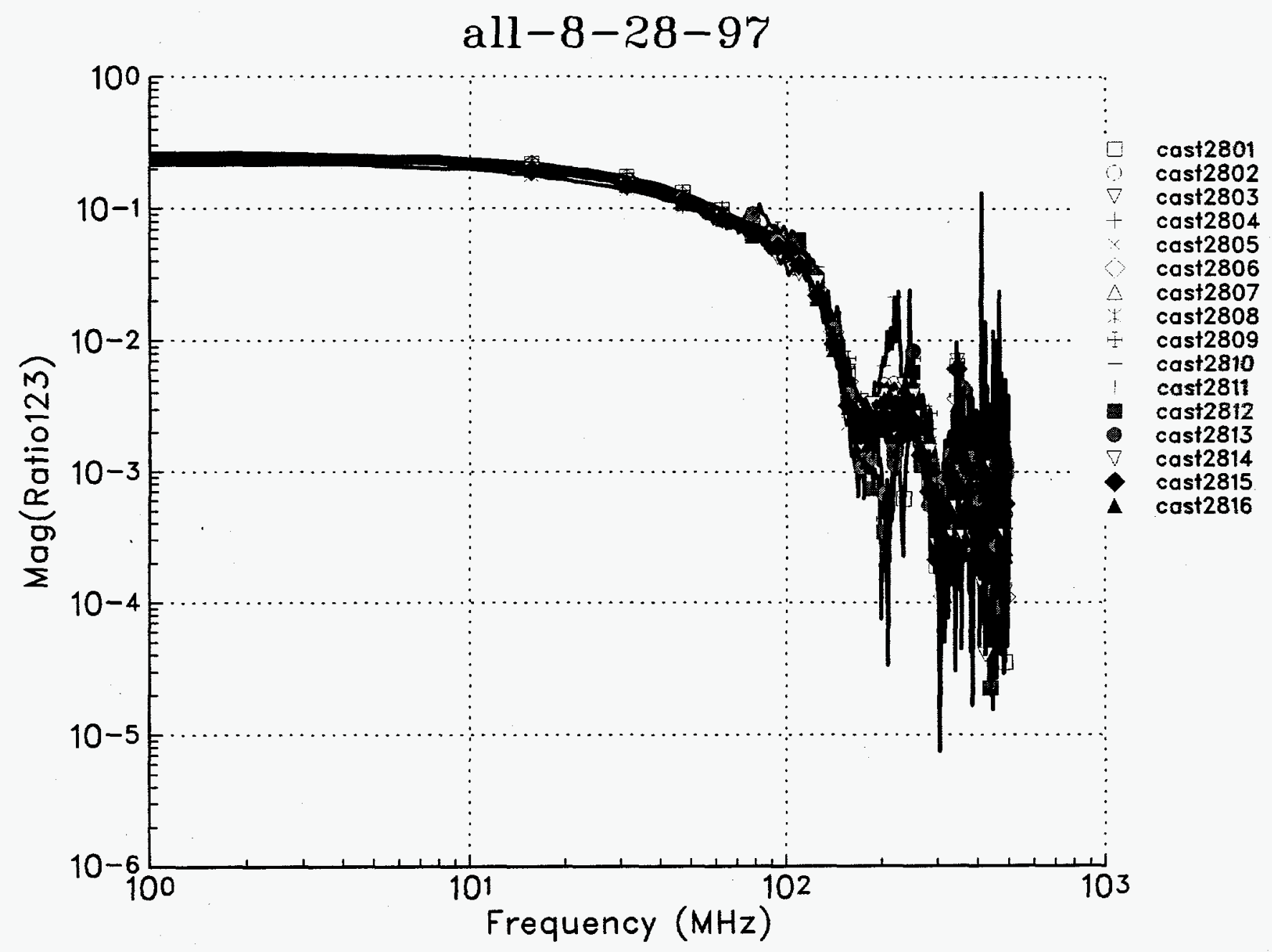

(13) 


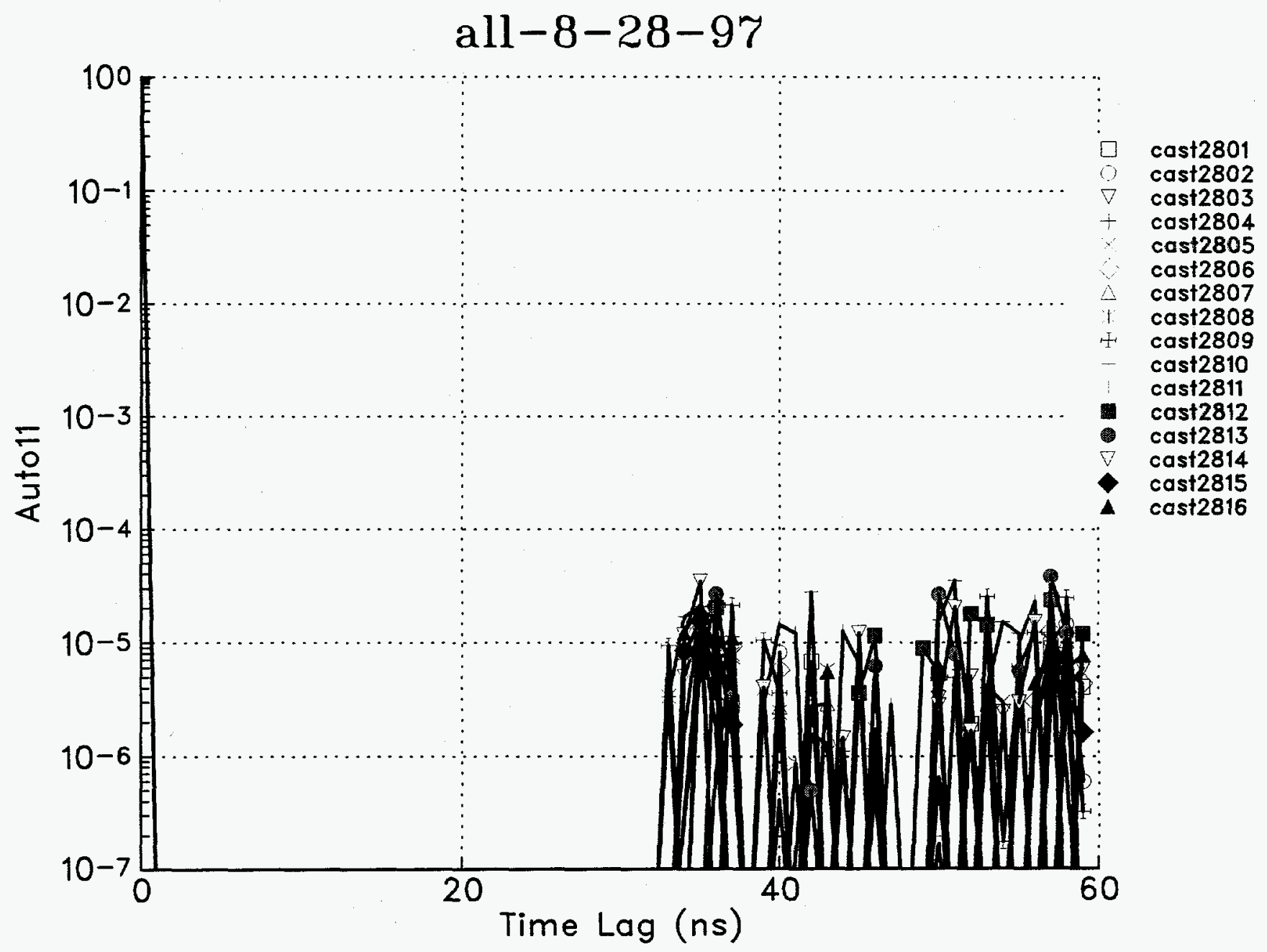

14 


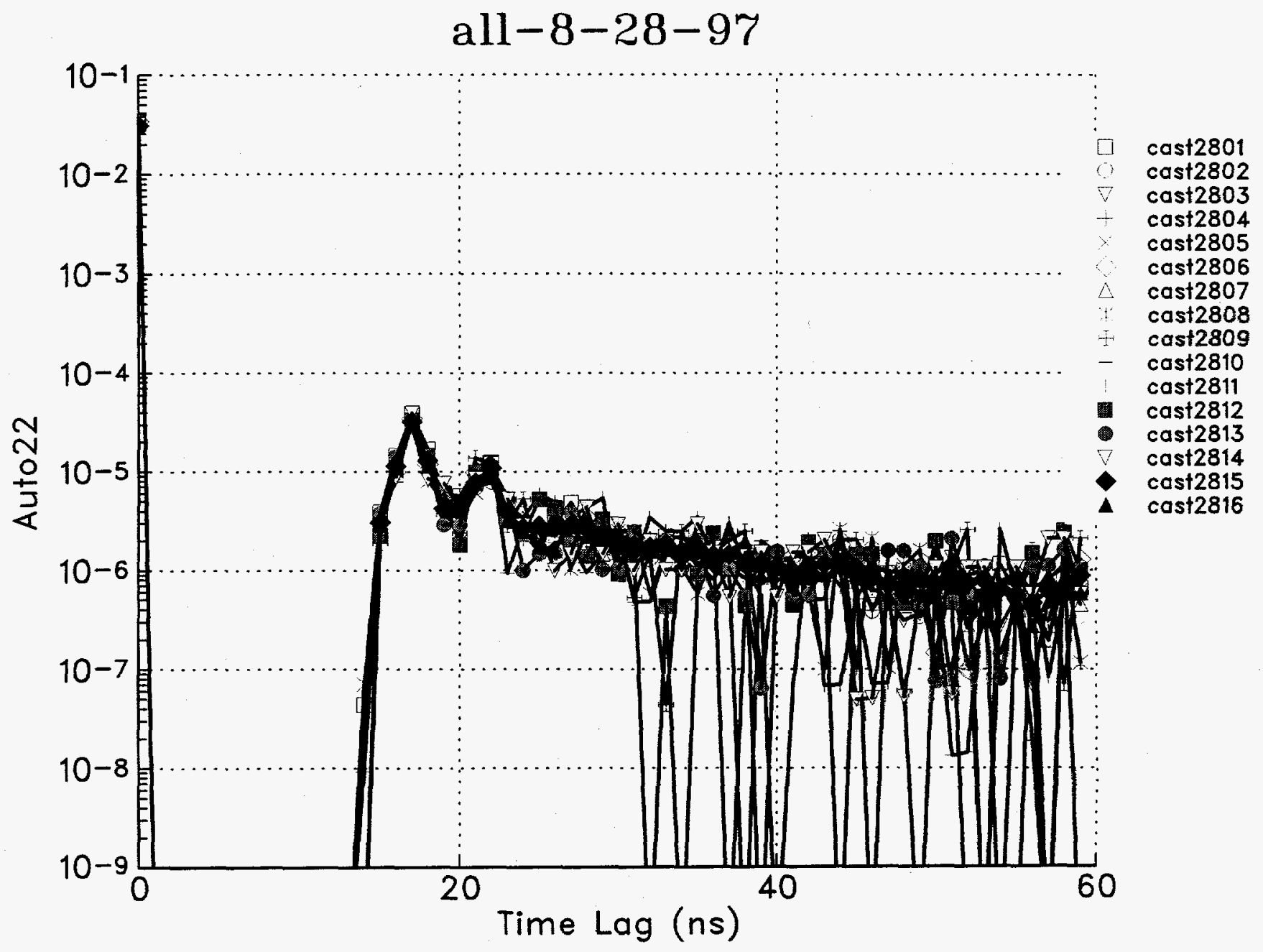

(15) 


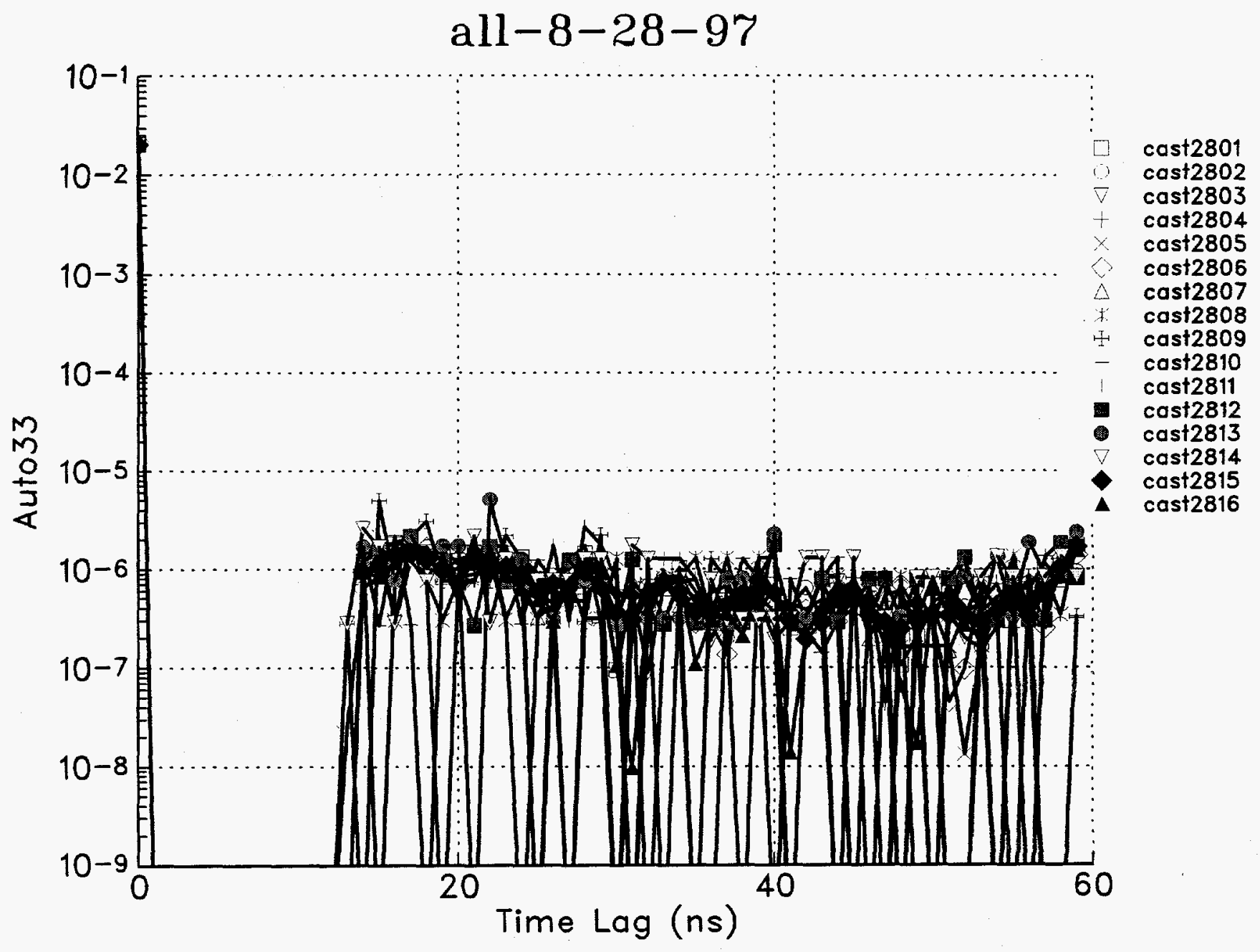

(16) 


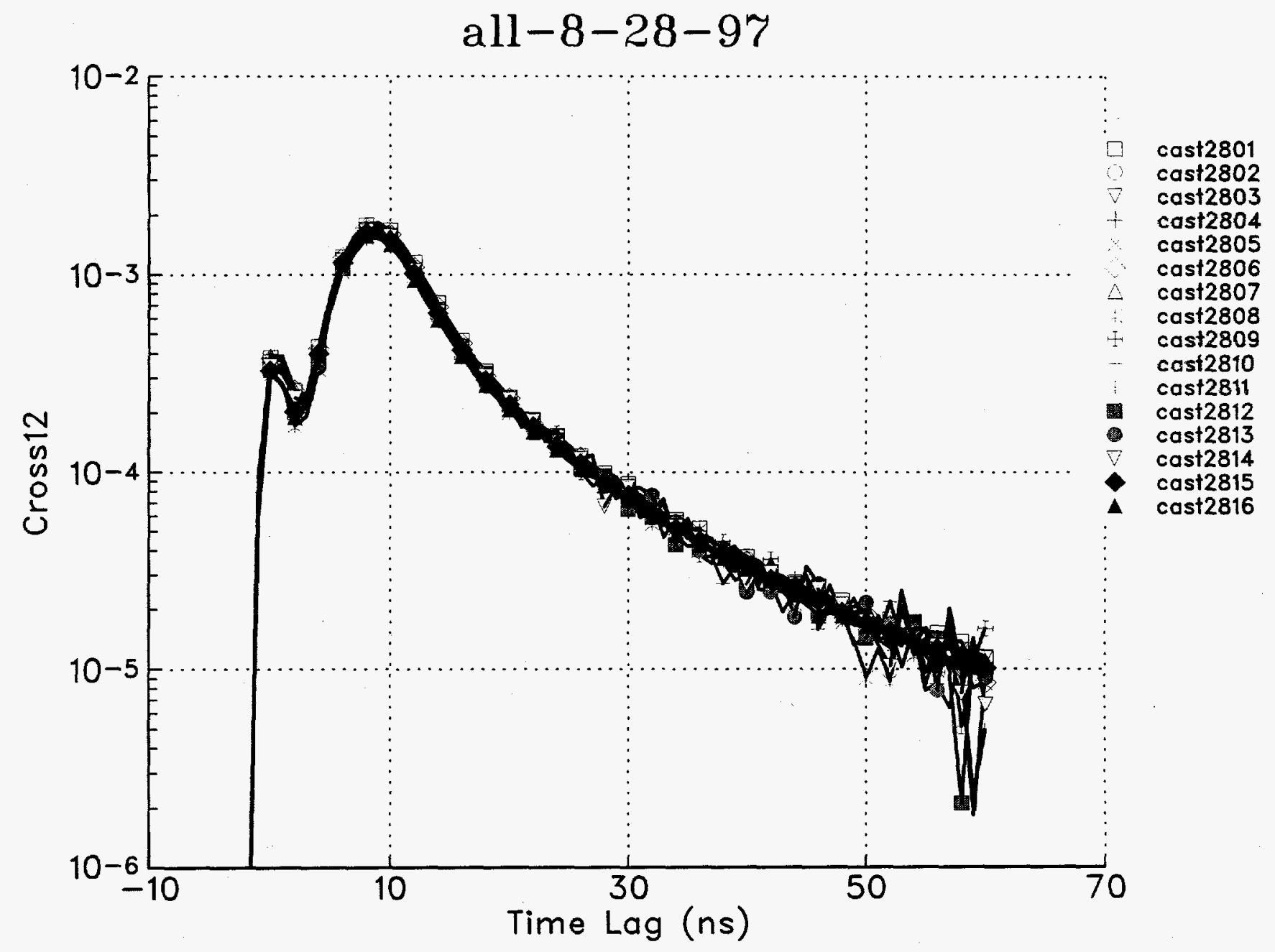

(17) 


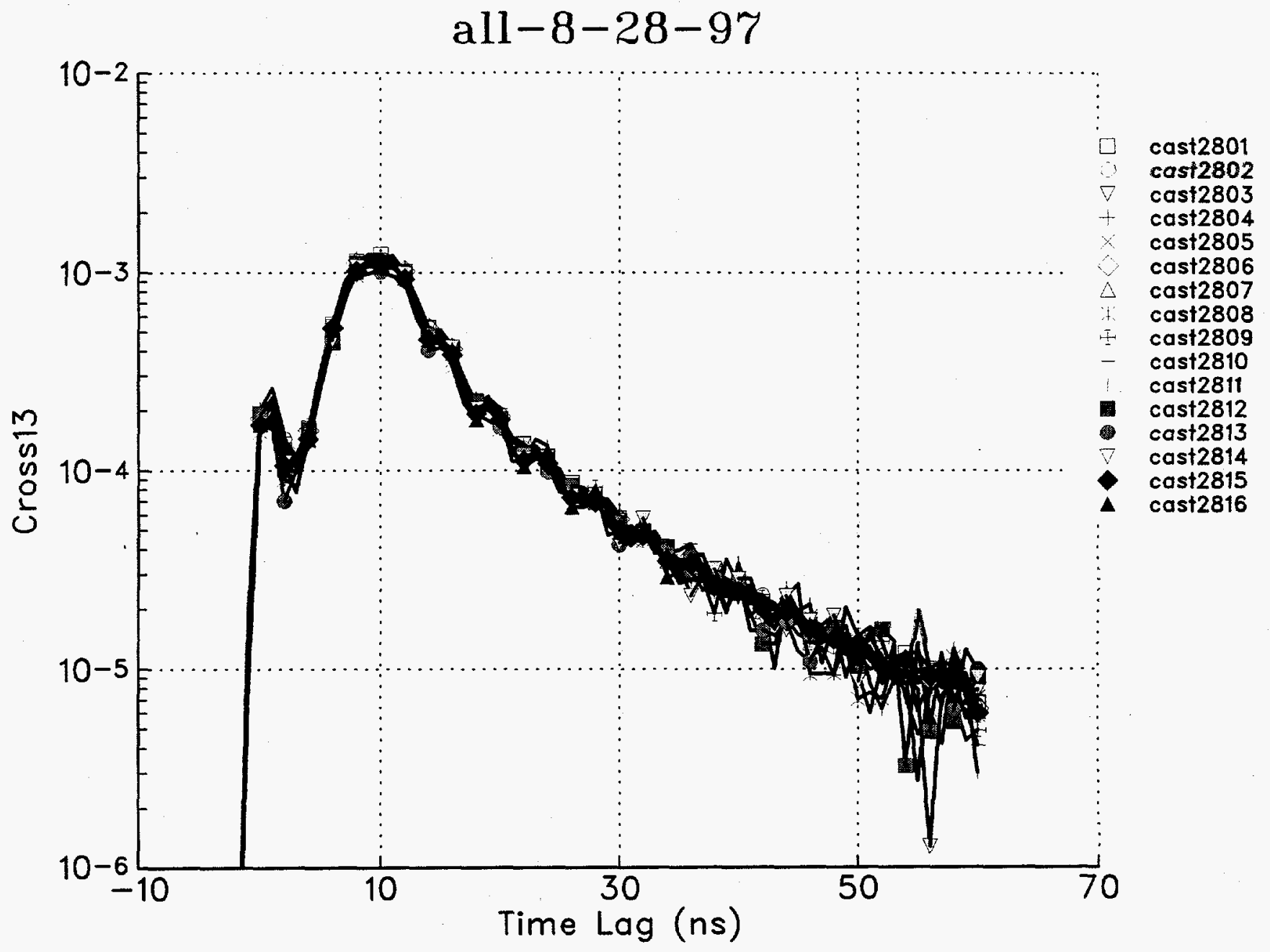

(18) 


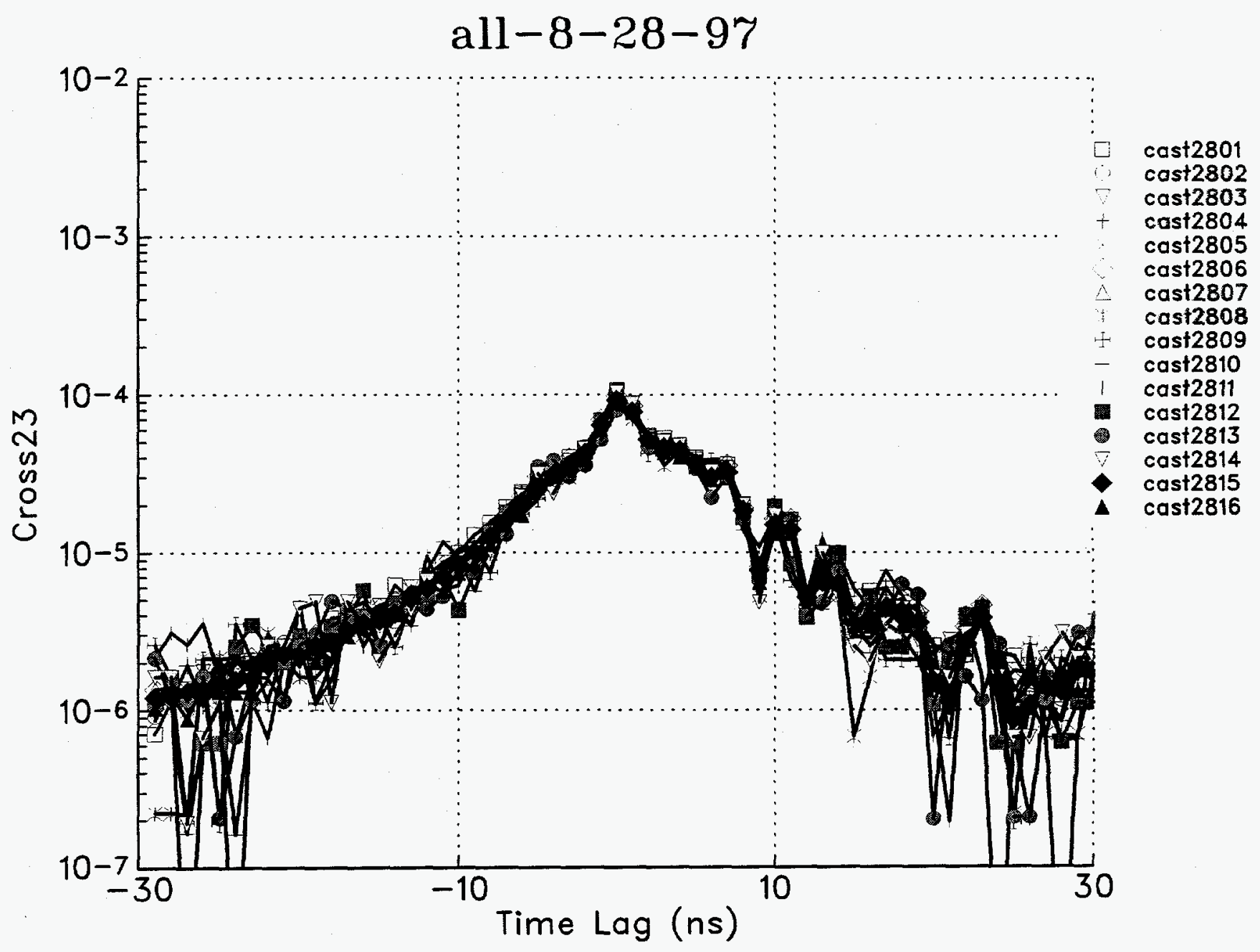

(19) 


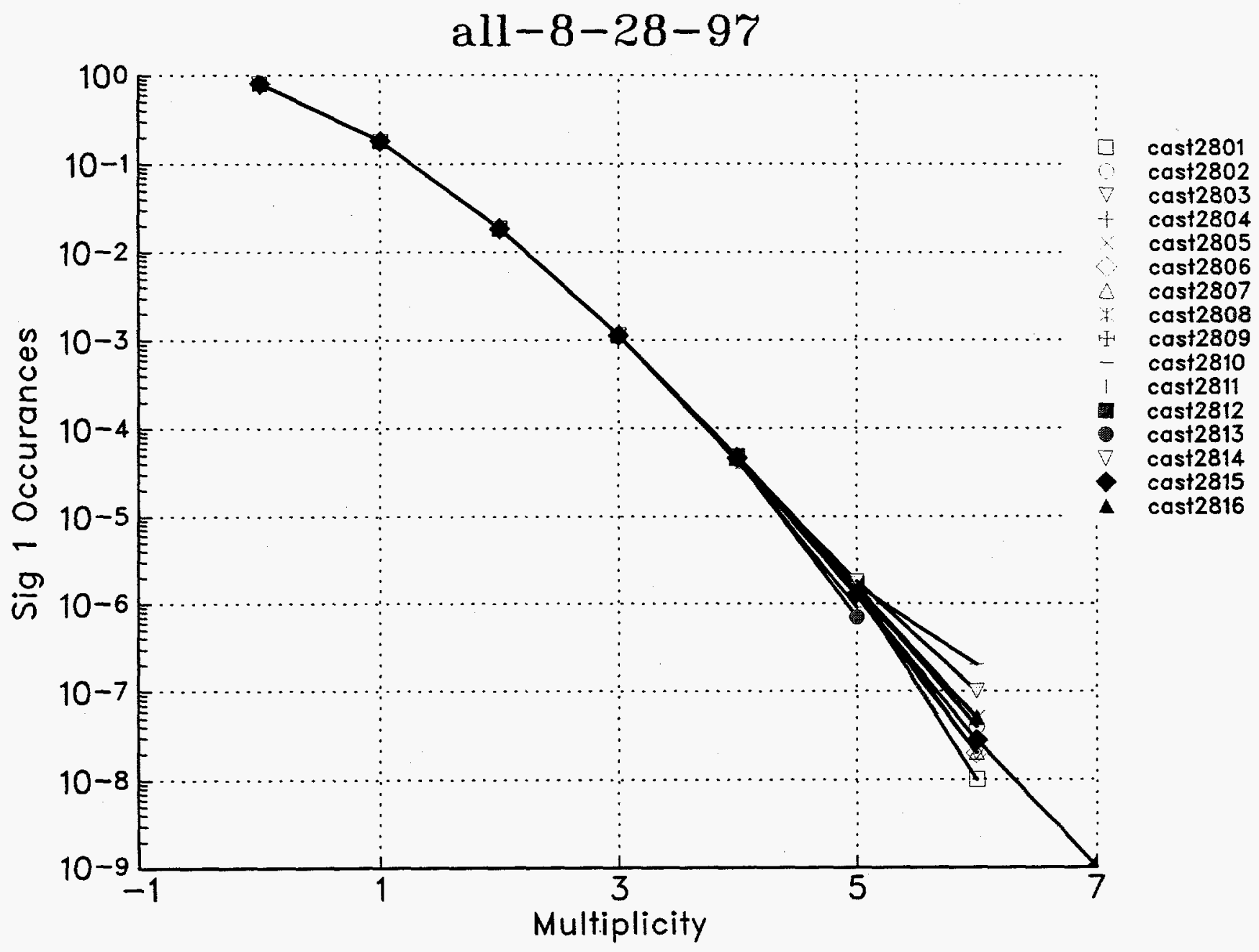

(20) 


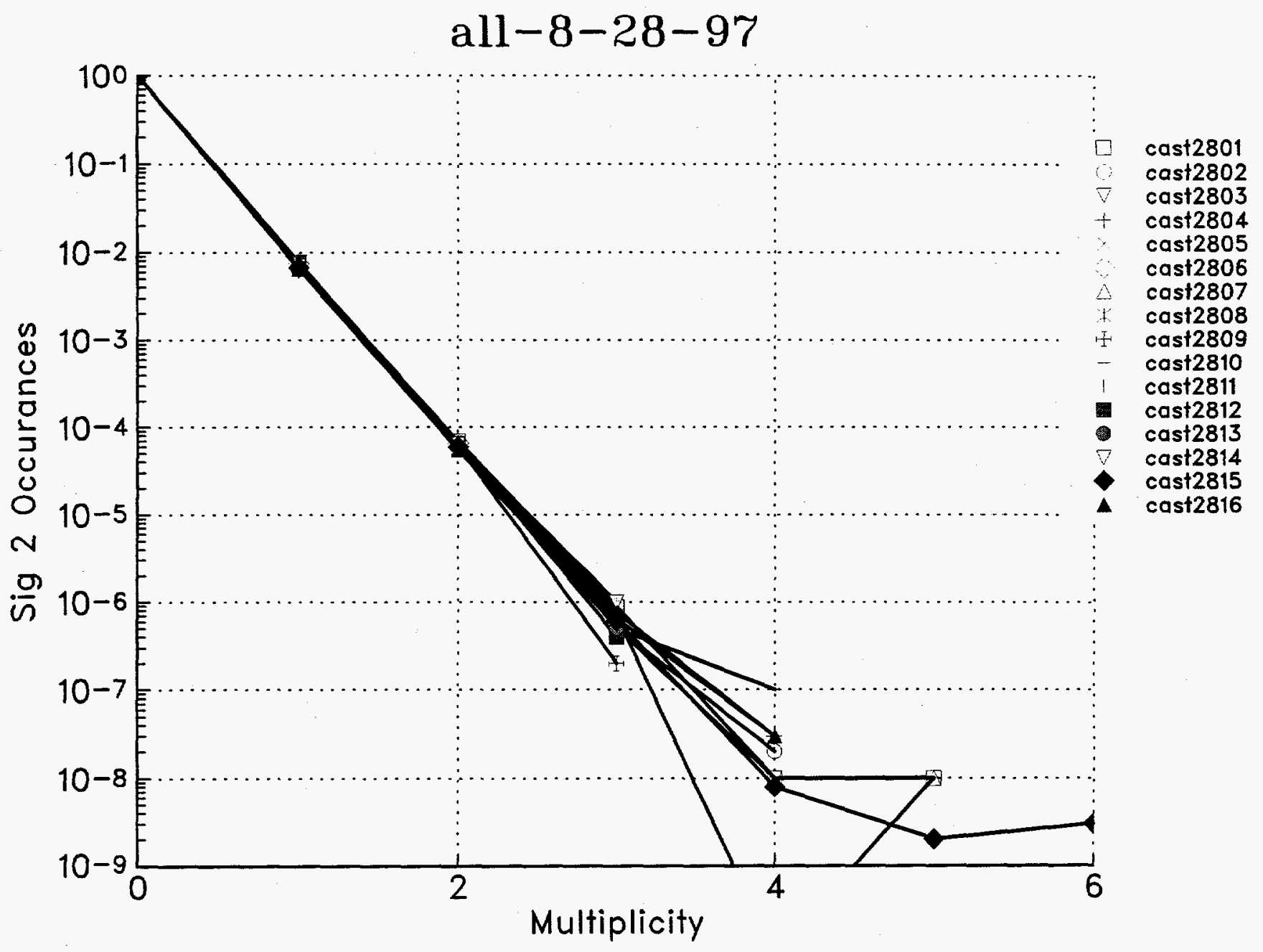

(21) 


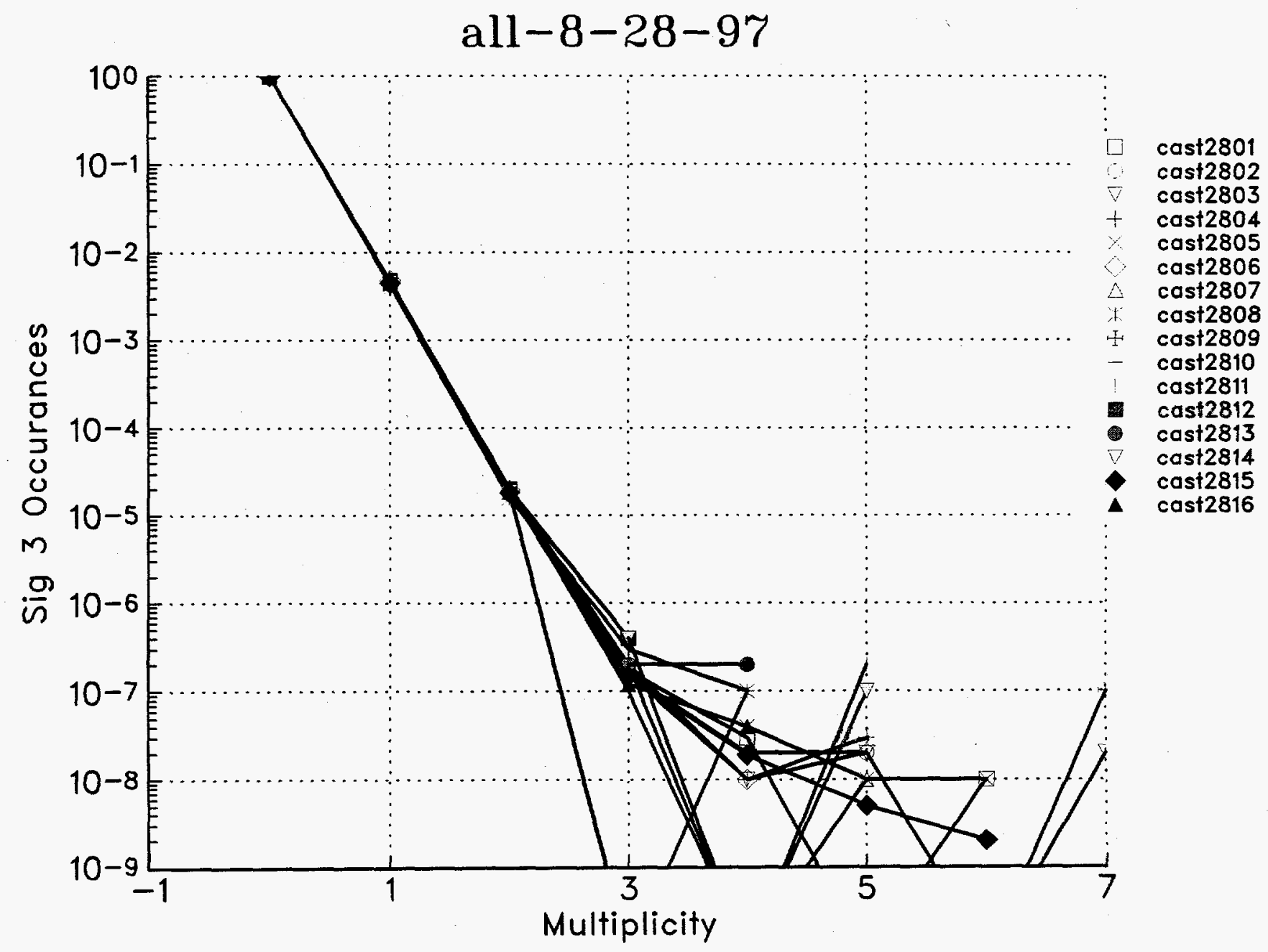

(22) 


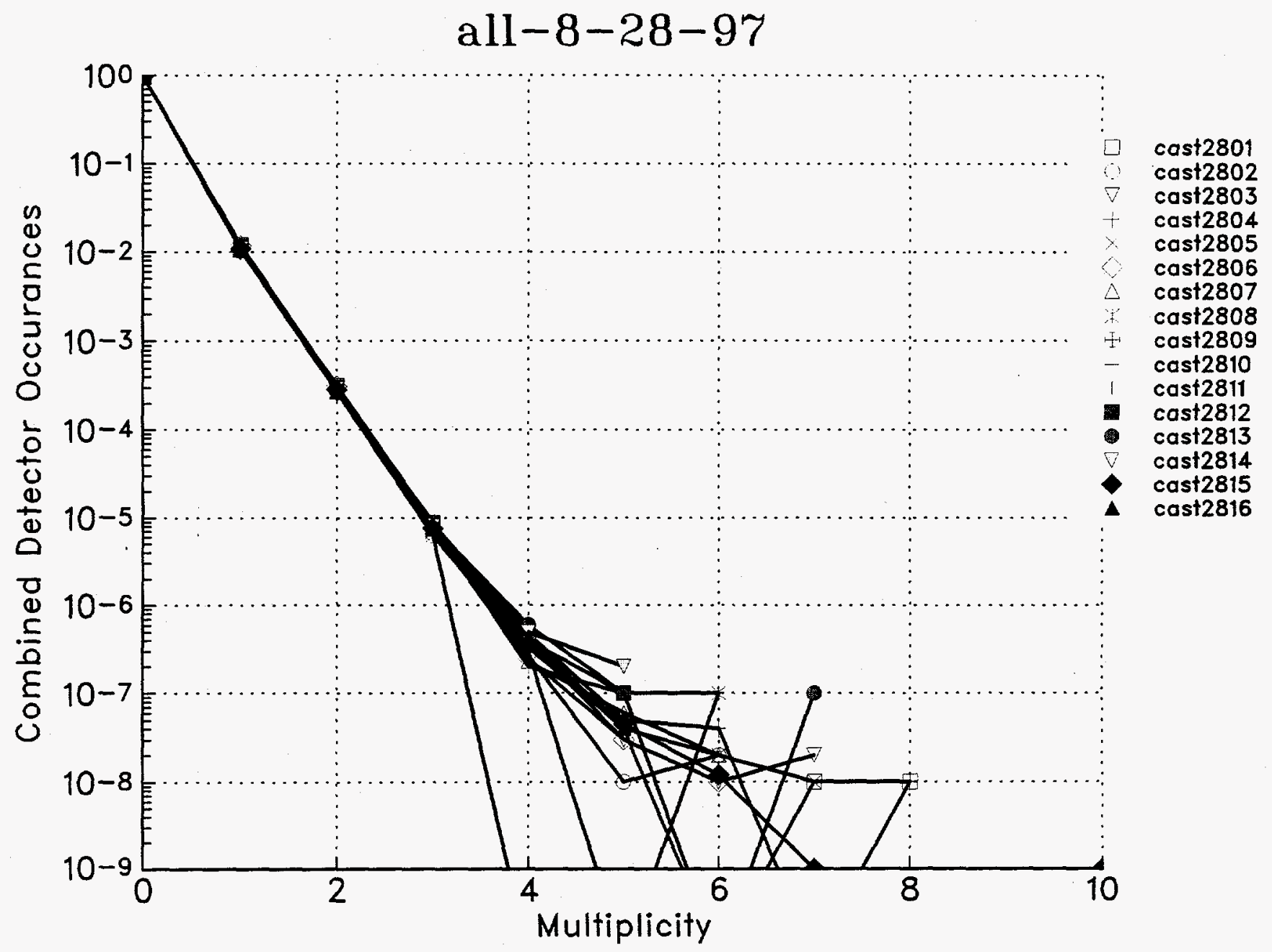

(23) 


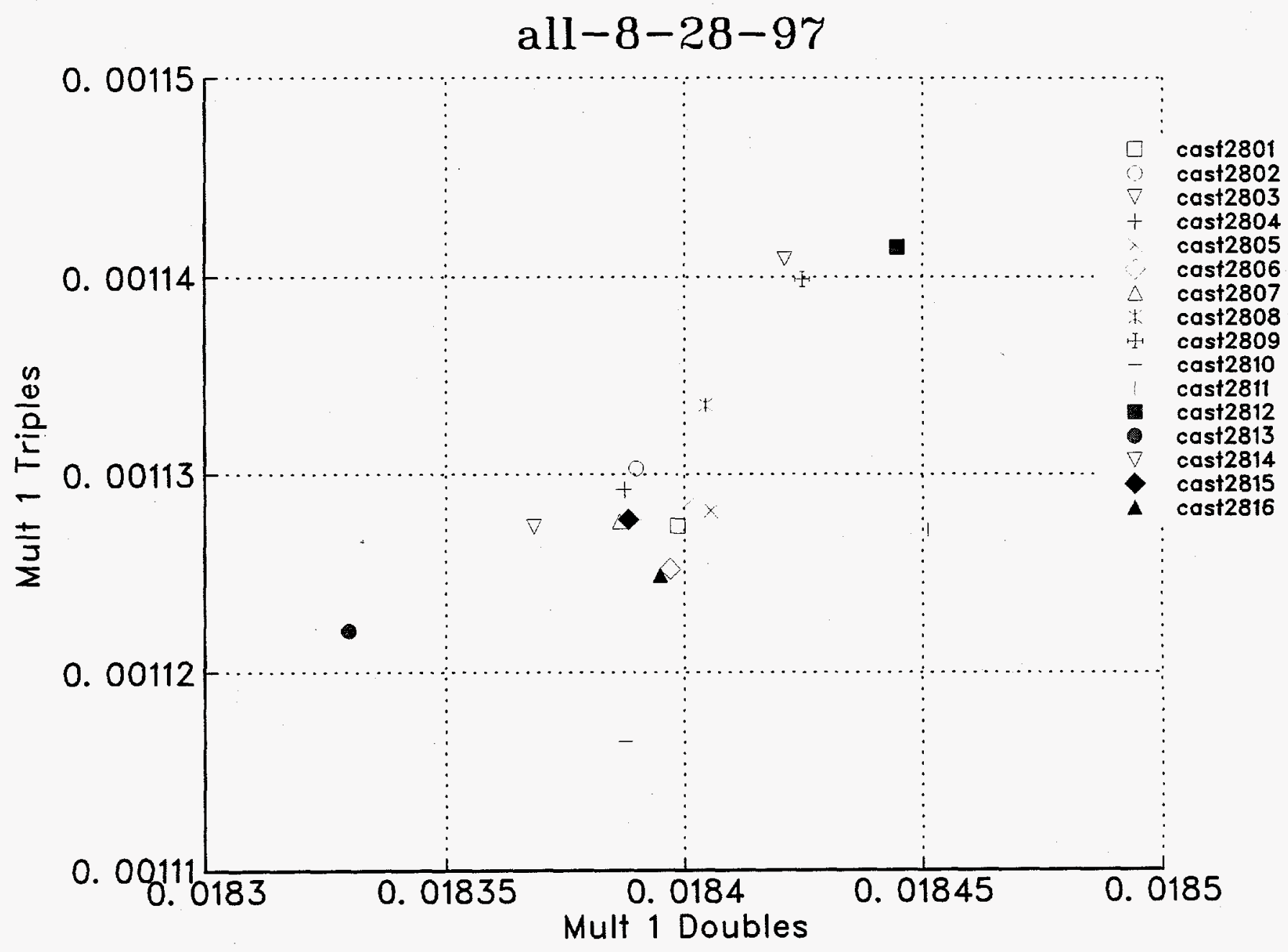

24 
Graphic 7.0 Apr. 14, 1998 1:36:43 PM

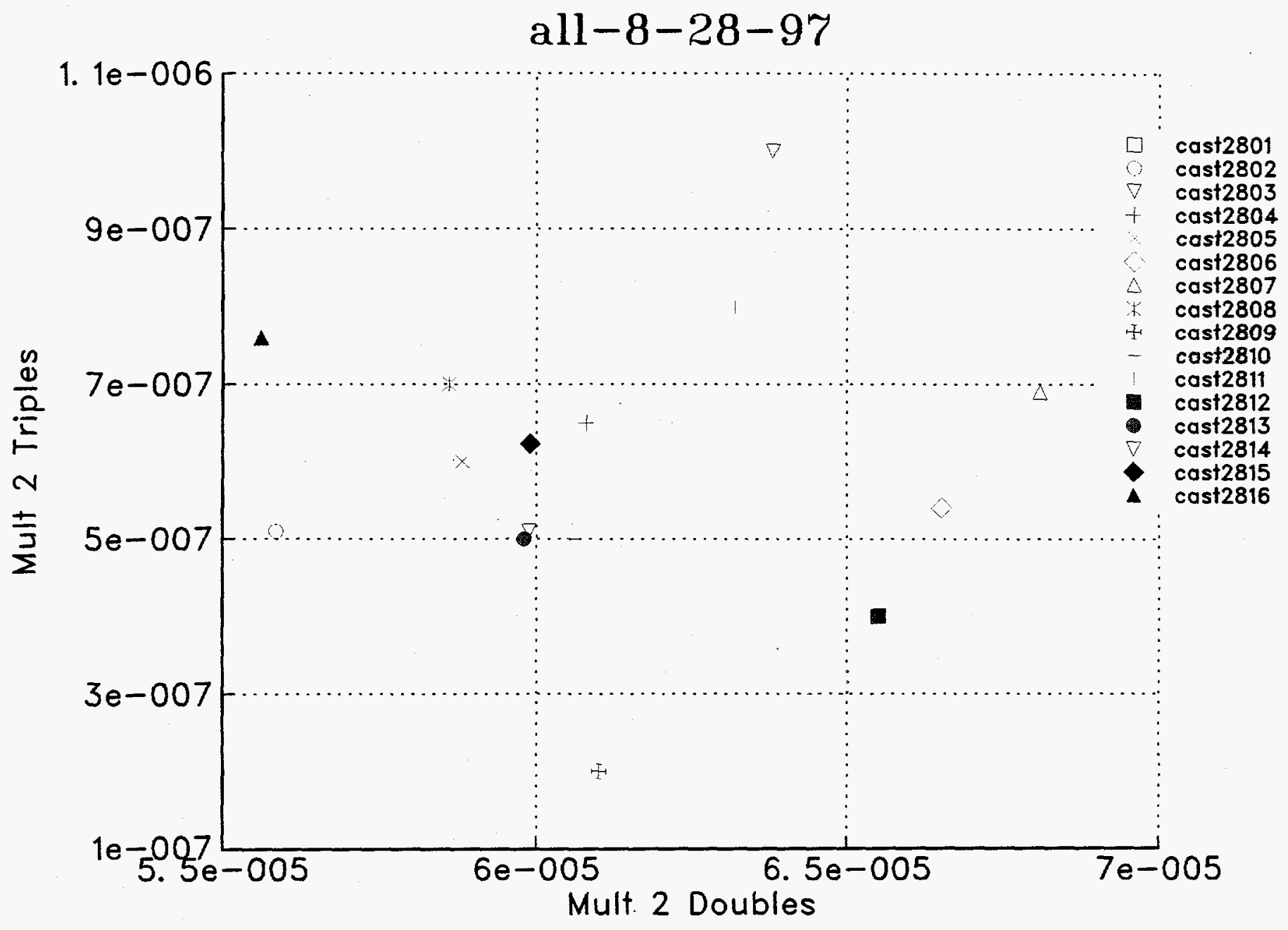

(25) 


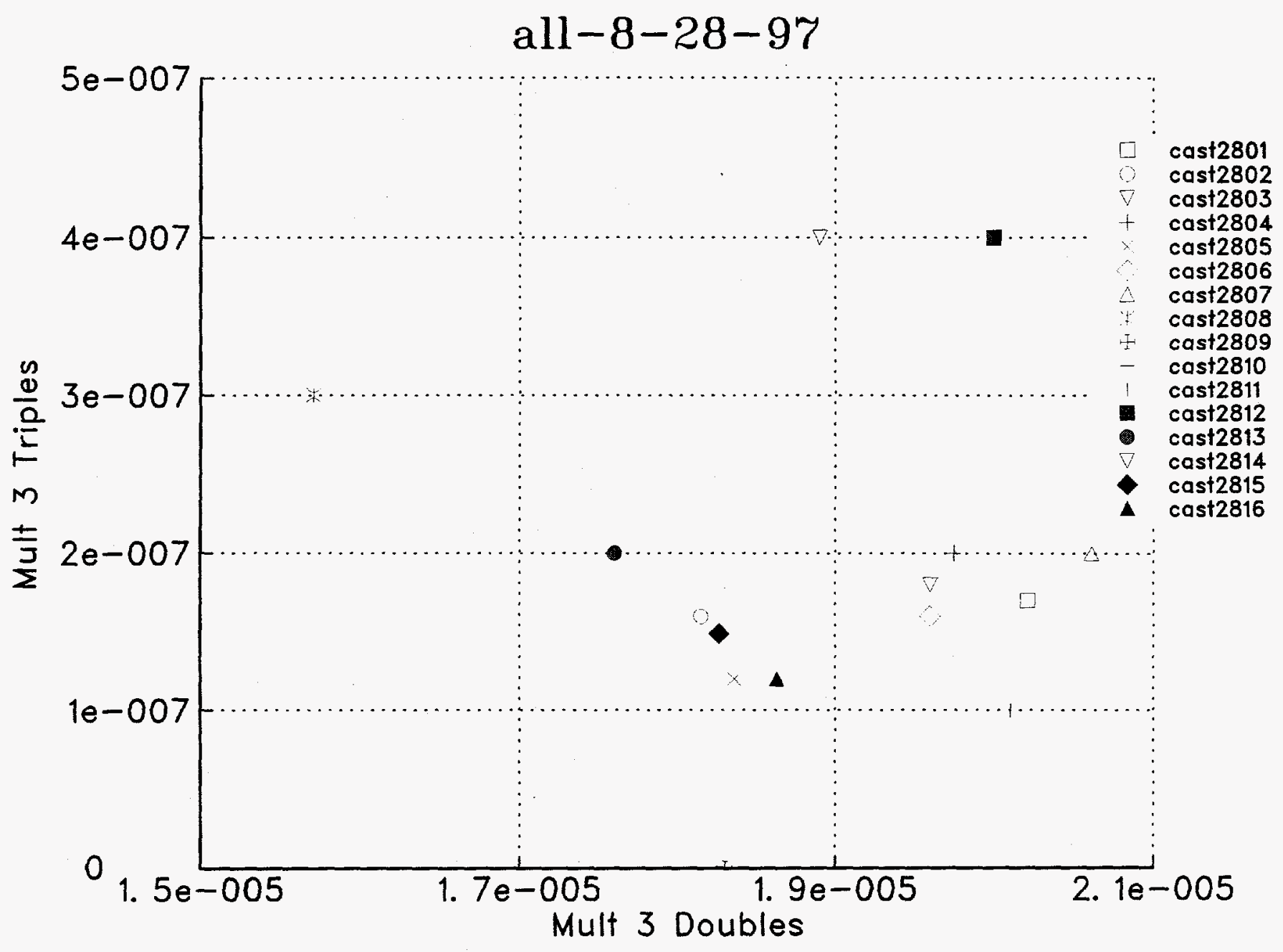

(26) 


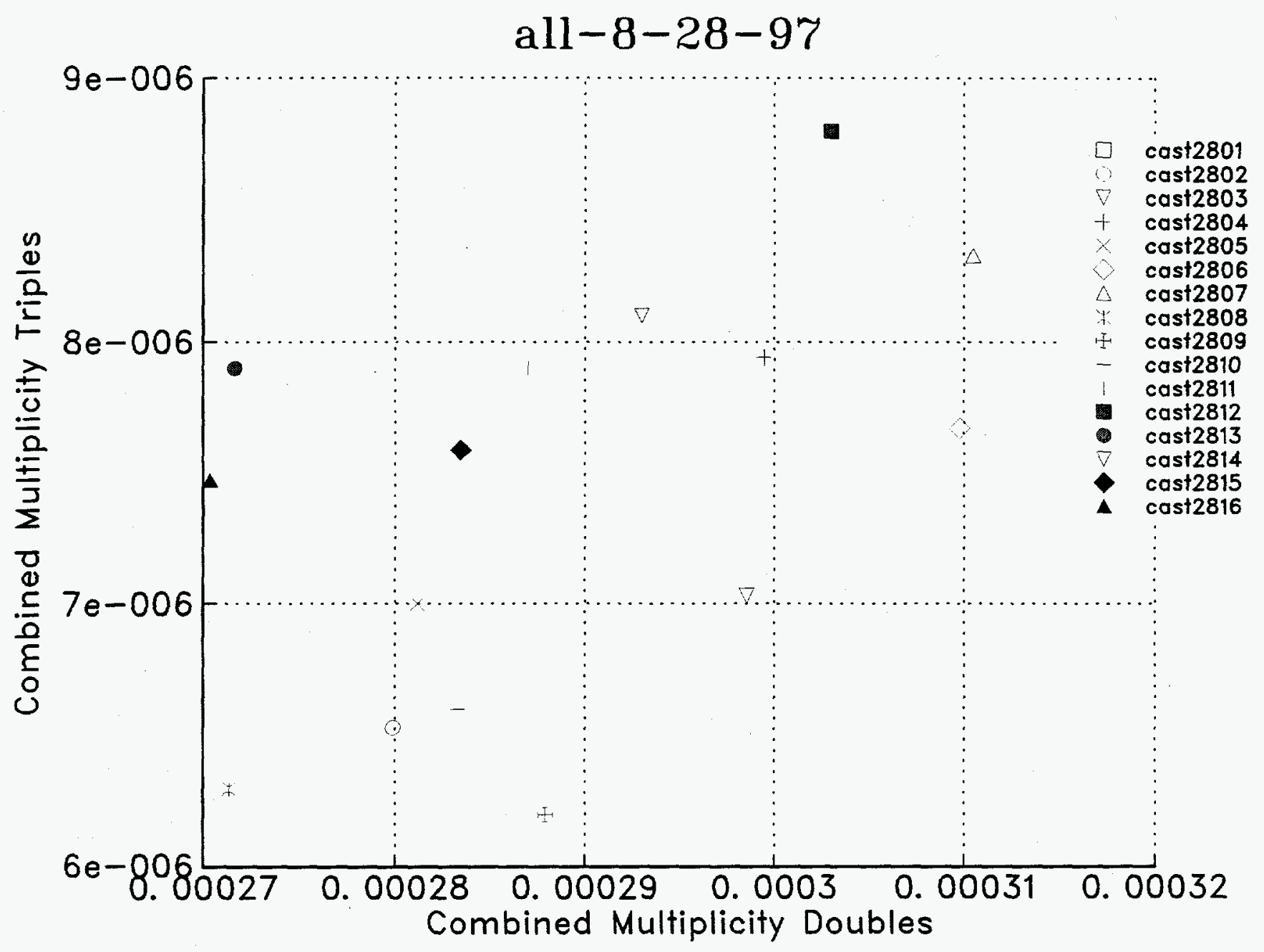

(27) 